\title{
Continuous time wavelet entropy of auditory evoked potentials
}

\author{
M. Emre Cek ${ }^{\mathrm{a}, *}$, Murat Ozgoren $^{\mathrm{b}}, \mathrm{F}$. Acar Savaci ${ }^{\mathrm{a}}$ \\ a Izmir Institute of Technology, Department of Electrical and Electronics Engineering, 35430 Urla, Izmir/Turkey \\ ${ }^{\mathrm{b}}$ Dokuz Eylul University, Faculty of Medicine, Department of Biophysics, Turkey
}

\section{A R T I C L E I N F O}

\section{Article history:}

Received 26 May 2008

Accepted 12 November 2009

\section{Keywords:}

Wavelet transform

Continuous wavelet entropy

Shannon entropy

Tsallis entropy

Relative wavelet energy

\begin{abstract}
A B S T R A C T
In this paper, the continuous time wavelet entropy (CTWE) of auditory evoked potentials (AEP) has been characterized by evaluating the relative wavelet energies (RWE) in specified EEG frequency bands. Thus, the rapid variations of CTWE due to the auditory stimulation could be detected in post-stimulus time interval. This approach removes the probability of missing the information hidden in short time intervals. The discrete time and continuous time wavelet based wavelet entropy variations were compared on non-target and target AEP data. It was observed that CTWE can also be an alternative method to analyze entropy as a function of time.
\end{abstract}

(c) 2009 Elsevier Ltd. All rights reserved.

\section{Introduction}

Since Hans Berger developed a non-invasive technique in 1929 to record Electroencephalography (EEG), the analysis of neural activities has been a challenging area in neuroscience. Basically, EEG is said to be a reflection of electrical activity related to neural structures in the brain which produce oscillations having different frequency ranges. Due to EEG signals having both a dynamic structure and non-stationary nature, the mathematical methods involving the analysis in time-frequency domain are essential to detect the hidden local information in neural activity. Wavelet analysis is a suitable means of analyzing these non-stationary signals, detailed theoretical formulation can be found in $[1,2]$.

While analyzing EEG signals including different states which contain mental activity, one has to take into account that a resonance occurs between neuron populations for finite time duration which indicates the existence of synchronization in specified frequencies [3]. The transition from disordered state to ordered state results in a single peak across the whole frequency band.

The conventional method to obtain the degree of disorder is defined by evaluating the spectral entropy obtained by the Fourier power spectrum [4]. Spectral entropy was also used for brain electrical signals by Inouye in [5,6]. The Fourier based entropy measures may lead to misleading results when applied to nonstationary signals. The EEG application is such an example where the spectral entropy would be insufficient to describe temporal

\footnotetext{
* Corresponding author. Tel.: +90232 750 6568; fax: +902327506599.

E-mail address: emrecek@iyte.edu.tr (M. Emre Cek).
}

information of underlying physiological activity. Since time evolution of state variations cannot be extracted by spectral entropy, then the short-time Fourier transform could be partially a candidate to illustrate to time-frequency representation of the evoked potentials [4]. But the constant size of time-frequency windows of short time Fourier transform (STFT) offers a limited resolution in both time and frequency domains. Therefore, wavelet based analysis has come into prominence due to flexible time-frequency atoms which are regarded as proper candidates to uncover the common mechanism of neural structures. The limitations associated with the time-frequency resolution may be overcome by the method given in [7-12] which is based on discrete time wavelet decomposition introduced as orthogonal discrete wavelet transform (ODWT) [1,13]. This method decomposes an observed signal into definite frequency bands without losing time evolution held by the wavelet coefficients. The relative wavelet energy is obtained by finding the ratio of the energy for each frequency band to the total energy in a given time interval. The wavelet entropy is then evaluated by using the normalized relative wavelet energies. Using a discrete wavelet transform approach, the event related potentials have been of interest to a number of studies, reporting the use of entropy functions in the electrophysiological data sets $15-16$.

This paper has been organized as follows. In the following section, the mathematical fundamental of continuous wavelet transform has been given. In section after, the definition of relative wavelet energies and the wavelet entropy which is characterized by Shannon entropy are explained. In this section, the experimental procedure and simulation results associated with auditory evoked potentials (AEP) are explained in detail. Finally, the performance of the continuous wavelet entropy based 
results is provided by illustrating the evolution of the wavelet entropy where the local minimum time instants of the continuous wavelet entropy could be detected at post-stimulus time interval.

The common approach in electrophysiological research is to sample data from a large group and use averages. This leaves behind a major problem, that is, the single subject data or single sweeps are overlooked. In order to overcome these problems, a method with proper resolution has to be incorporated in this prospect. Accordingly, this paper provides a balanced scope with the utilization of CWTE. The general conclusions may carry a risk of overfitting, which can be ruled out with further applications of this method to different data sets.

\section{Methods}

\subsection{Wavelet transform}

The continuous time wavelet transform converts a one dimensional function $s(t)$ which corresponds to the observed electrical activity into two dimensional function represented by scale and dilation parameters. This is shown as below [1]

$\boldsymbol{W}_{\boldsymbol{s}}(\boldsymbol{a}, \boldsymbol{t})=\int_{-\infty}^{\infty} \boldsymbol{s}(\tau) \psi\left(\frac{\tau-\boldsymbol{t}}{\boldsymbol{a}}\right) d \tau$

where $\psi(\cdot)$ is the mother wavelet, a vanishing function fast decaying to zero in both time and frequency domain. The wavelet transform is computed by using time and scale parameters while the transformed data are illustrated commonly in terms of time and frequency. Therefore the conversion from a given scale $a$ to the corresponding frequency $f$ can be used with the given formulation by (2)

$\boldsymbol{f}=\frac{\boldsymbol{F}_{\boldsymbol{c}} \cdot \boldsymbol{F}_{\boldsymbol{s}}}{\boldsymbol{a}}$

where $F_{s}$ is the sampling frequency and $F_{c}$ is the center frequency of the selected mother wavelet. By this conversion, the wavelet coefficients can now be represented in terms of time-frequency $W_{s}(t, f)$. The detailed information about the relation between scale and frequency can be found in [14]. In order to explore the time evolution of the total wavelet entropy, these wavelet coefficients represent the sliding time windows with full overlapping over time.

\subsection{Relative wavelet energy}

Relative wavelet energy (RWE) is expressed as the summation of wavelet coefficients within a selected frequency range over the time. The RWE was obtained by continuous wavelet transform coefficients, with an approach differing from the previous analysis given in [15-17]. Wavelet entropy has been evaluated by using continuous wavelet transform coefficients as in the previous works $[18,19]$. Since EEG signals of length $N$ are sampled at $t_{n}=n T_{s}, n=1,2, \ldots, N$ with a sampling period $T_{s}=1 / F_{s}$ the formulation related with wavelet energy in the frequency interval $\left[f_{1} f_{2}\right]$ is given in terms of summation of the sampled values of wavelet energy coefficients as given below

$E_{\left[f_{1} f_{2}\right]}=\sum_{m=0}^{\left(f_{2}-f_{1}\right) / \Delta f} \sum_{n}\left|W_{s}\left(t_{n}, f_{1}+m \cdot \Delta f\right)\right|^{2}$

where $\Delta f$ is the step size of the frequency. The total wavelet energy as a function of time in each time window $w$ can be expressed as

$E_{\text {tot }}\left(t_{n}\right)=\sum_{j} E_{\left[f_{j} f_{j+1}\right]}\left(t_{n}\right)=\sum_{j}\left\{\sum_{m=0}^{\left(f_{j+1}-f_{j}\right) / \Delta f} \sum_{k=0}^{w / T_{s}}\left|W_{s}\left(t_{n}-\frac{w}{2}+k T_{s}, f_{j}+m \cdot \Delta f\right)\right|^{2}\right\}$

The investigated frequency spectrum is bounded within $1-48 \mathrm{~Hz}$ and divided into subbands as given in (3) which are used to evaluate probability distribution. The term $\left[f_{j} f_{j+1}\right]$ corresponds to delta $(1-4 \mathrm{~Hz})$, theta $(4-8 \mathrm{~Hz})$, alpha $(8-13 \mathrm{~Hz})$, beta $(13-32 \mathrm{~Hz})$ and gamma $(32-48 \mathrm{~Hz})$ bands, respectively, where $j=1, \ldots, 5$ providing that $f_{1} \in\left[f_{1} f_{6}\right]$. In Eq. (4), the total energy of the signal is used to normalize each of the individual wavelet energies $E_{j}\left(t_{n}\right)=E_{\left[f_{j} f_{j+1}\right]}\left(t_{n}\right)$ which correspond to the energy localized in a specified frequency band. The relative wavelet energy can be obtained by computing the probability of wavelet energy observed within a frequency band $\left[f_{j} f_{j+1}\right]$ that can be expressed as

$p_{j}\left(t_{n}\right)=E_{j}\left(t_{n}\right) / E_{\text {tot }}\left(t_{n}\right)$.

\subsection{Wavelet entropy}

Analysis of probability distributions obtained in Section 2.2 is used to characterize the Shannon entropy [20]. The total wavelet entropy (WE) is a measure which gives information about the complexity of the signal. The formulation of Shannon WE is defined in [15] as

$W E_{S}=-\sum_{j} p_{j} \ln \left(p_{j}\right)$

and the Tsallis wavelet entropy is given in Eq. (7) as

$W E_{T}^{q}=\frac{1}{q-1} \sum_{j}\left(p_{j}-p_{j}^{q}\right)$

The signal having components at all frequency bands exhibits more disordered behavior and consequently the wavelet entropy has a higher value. In contrast any frequency band becomes dominant among other frequencies after stimulation is applied and the neural activity passes through an ordered state which causes the entropy to decrease. In other words, the concentration of frequency spectrum over some narrow frequency bands indicates the increase of periodicity, therefore lower entropy is observed for bounded time duration.

\subsection{Experimental setup}

The sample data were acquired from Dokuz Eylul University Brain Biophysics lab records. The data were acquired using Neuroscan 4.3 system and 64 EEG amplifier. The subject was a 22 year old male, a right handed medical student with no known history of neurological and psychological disorders. The stimuli were auditory tones of 1500 and $1600 \mathrm{~Hz}$ with a random ISI of $3.5-6 \mathrm{~s}$. The $1500 \mathrm{~Hz}$ stimuli were given as non-target and $1600 \mathrm{~Hz}$ stimuli were the target ones with a random probability of appearing about 20 percent in the sequence (app. non-target 120 ; target 30 sweeps). The auditory stimuli were administered via loudspeakers at an intensity of $80 \mathrm{dBL}$. The data consisted of 27 sweeps with $2 \mathrm{~s}$ duration. The subject was required to mentally count the target tones and report at the end of the session. The targets were correctly counted and reported by the subject. The EEG data sampling was $1000 \mathrm{~Hz}$. The sweeps were epoched using an analysis program of Scan 4.3 and MATLAB. The artifacts (eye blink, etc.) were removed visually, offline. For the comparative analysis the sweep numbers were equated to 27 . For the sake of simplicity, only central $(\mathrm{Cz})$ electrode information is provided in the analysis and figures. Two time windows were selected for the 
analysis of pre-stimulus and post-stimulus for both target and non-target entropy evaluation: $200-400 \mathrm{~ms}$ for the pre-stimulus as the first time window, for the post-stimulus, $50-200 \mathrm{~ms}$ as the second time window and $\mathbf{3 5 0 - 5 0 0 ~} \mathrm{ms}$ as the third time window. The respective peaks of entropy were evaluated by means of a MATLAB routine, reporting the mean entropy values associated with these three time intervals for every single sweep, both for the target and non-target stimuli responses. Normality of data was verified with the Kolmogorov-Smirnov test. As only two conditions were (target vs. non-target) planned to be compared, paired sample $T$ test was applied. The paired $t$-test was applied as the statistical test to compare the differences between the entropy values.

\section{Results}

The signals acquired by AEP were analyzed by continuous time wavelet transform where the mother wavelet was chosen as Bspline basis function as recommended in [21-23]. Improper selection of center frequency and bandwidth related with the mother wavelet would cause in a decrease of the resolution in both time and frequency therefore detailed analysis for the proper selection of mother wavelet which is given for auditory evoked potentials in [23]. The time domain evoked potentials of averaged 27 sweeps for non-target and target are given with their continuous wavelet transforms in Fig. 1, respectively. The wavelet time-frequency distribution was illustrated according to the energy levels in Fig. 1.

The continuous time evolution of the wavelet energies is illustrated in Fig. 2. It can be easily seen that the energy of each frequency band significantly increases at post-stimulus time interval. Since the magnitude of the wavelet energy is relatively low for the gamma band, it was not illustrated in Fig. 2. The wavelet energies related to delta and theta band strongly increases in post-stimulus time interval, contributing mainly to the decrease of entropy.
The paper summarizes the time evolution of Shannon wavelet entropy and the relative wavelet energies over the auditory evoked potentials. Wavelet entropy is represented in this paper by using Shannon entropy so that the variations of local wavelet energy distributions can be exhibited in the time-frequency domain.

As shown in Fig. 3, both methods exhibit almost the same performance for non-targets (Fig. 3a) but it is noticeable that entropy variation in early post-stimulus range (0.05-0.2 s) may not be detected if a wider time window was chosen for targets (Fig. 3b). One can also detect that time evolution of the discrete
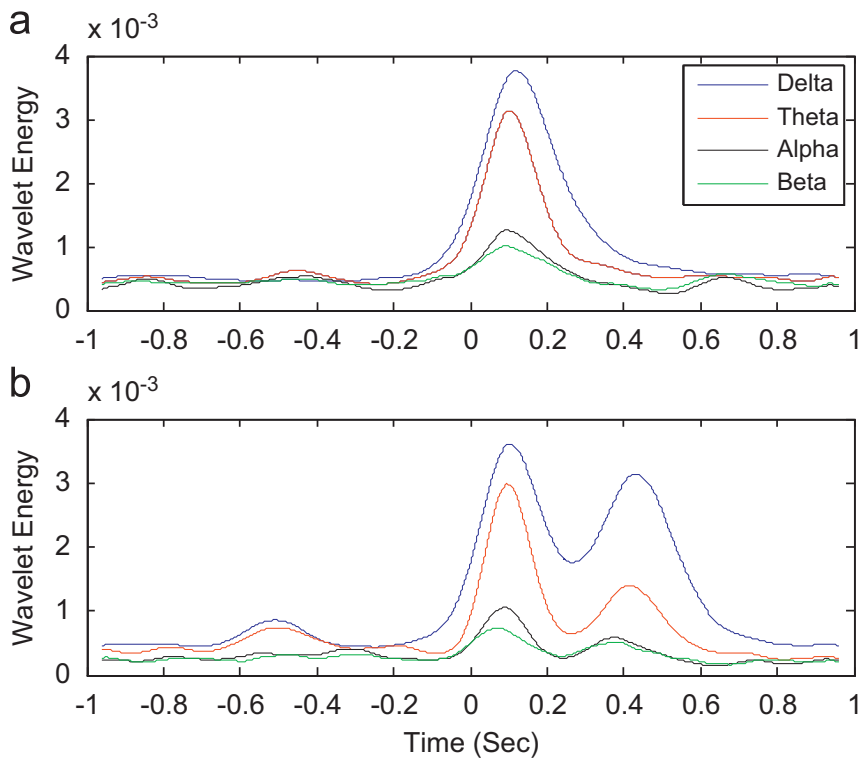

Fig. 2. The wavelet energy of each frequency band as a function of time of auditory evoked potentials is shown for non-targets (a) and target (b). The $x$-axis is the time axis where the stimulus is applied at time instant " 0 ", the $y$-axis gives the amplitude of the wavelet energies.
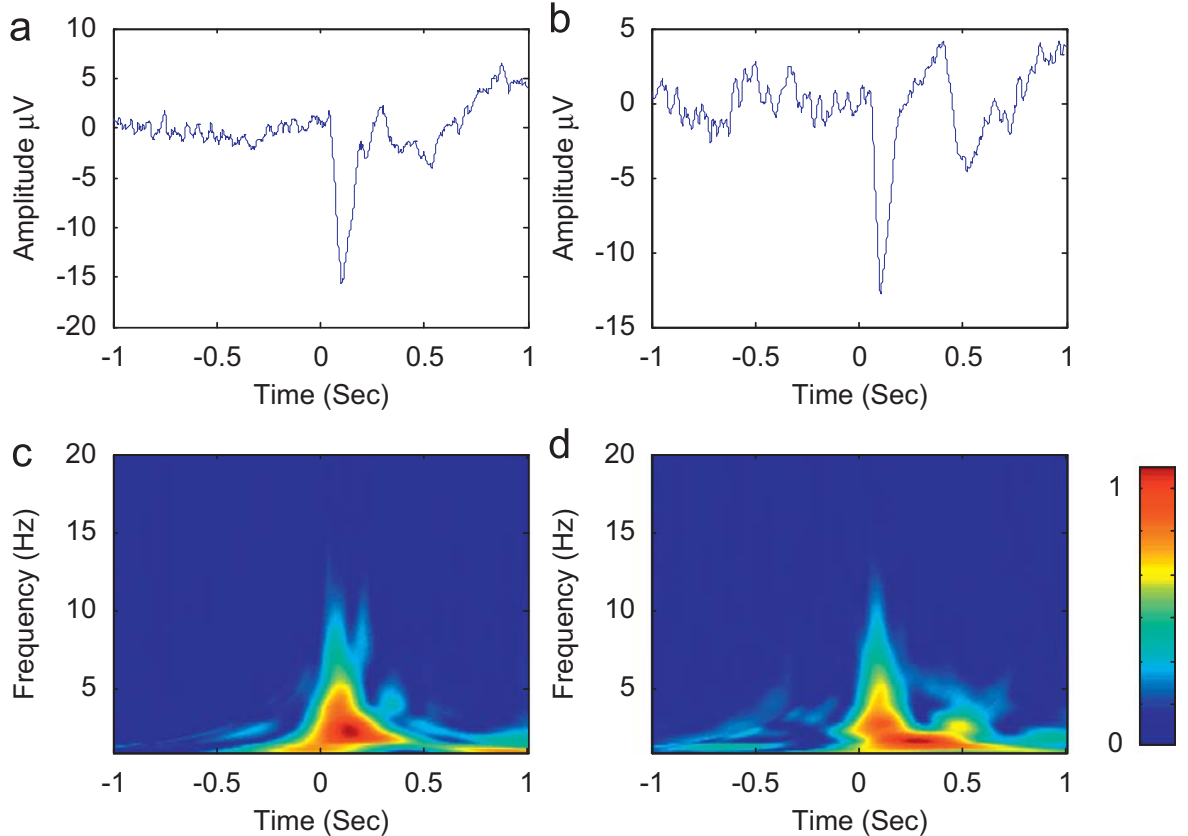

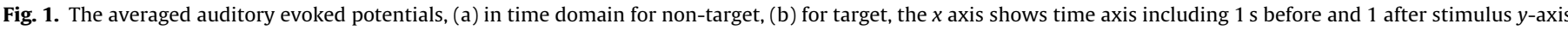

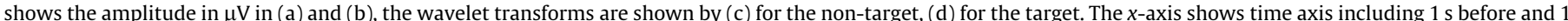
after stimulus and $y$-axis corresponds to frequency in $\mathrm{Hz}$ in (c) and (d). The color bar denotes the normalized wavelet energies for (c) and (d). 
a

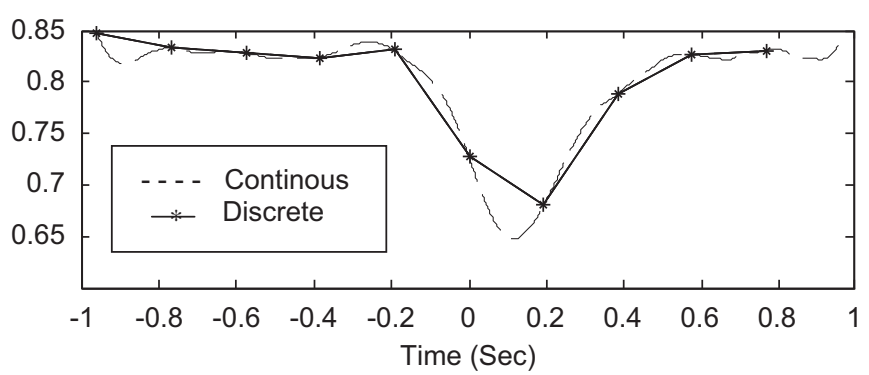

b

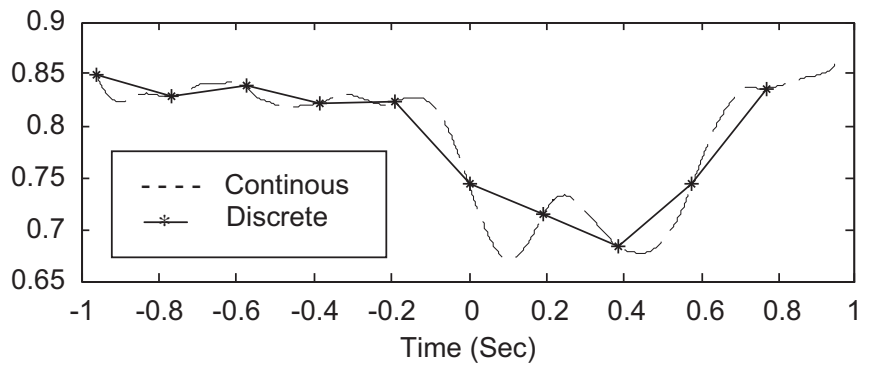

Fig. 3. The representation of time dependent continuous (solid) and discrete wavelet entropy (dashed) for non-targets (a) and targets (b) are given where the non-overlapping window size associated with discrete wavelet transform was chosen as $192 \mathrm{~ms}$. The $x$-axis gives the time interval having $1 \mathrm{~s}$ pre-stimulus and $1 \mathrm{~s}$ post-stimulus. The $y$-axis gives the normalized entropy value. It can be noticed that discrete wavelet transform based method misses the abrupt changes especially in post-stimulus time intervals.

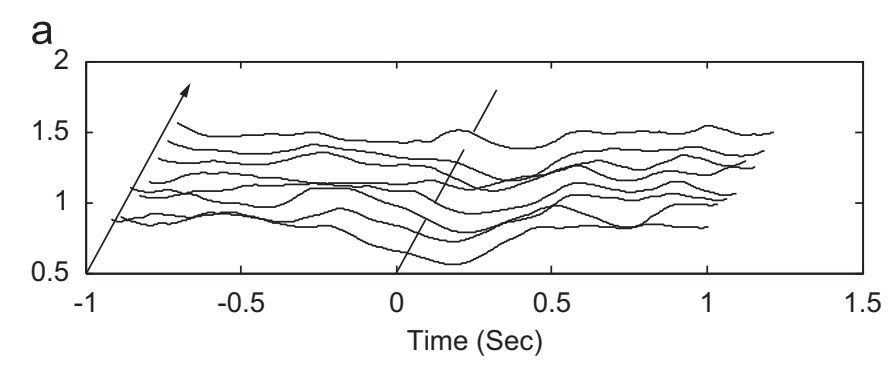

b

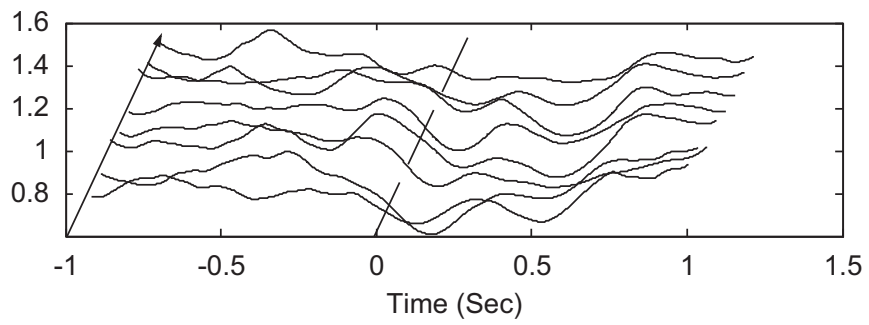

Fig. 4. The representation of the time dependent wavelet entropy for each sweep is given for (a) non-target and (b) target responses. The evolution of the wavelet entropy in post-stimulus time interval differs for non-target and target sweeps.

time wavelet entropy based method misses the lower peak between 0.35 and $0.5 \mathrm{~s}$ time intervals, while continuous wavelet entropy based method represents the exact location of the lower peak. In this work it is noticeable that continuous wavelet transform based time-dependent wavelet entropy may provide more detailed results including abrupt changes in entropy.

Additionally, we provide a single sweep entropy analysis demonstrating the temporal evolution. This is a novel presentation of the ERP data, indicating the sensitivity of the proposed continuous wavelet entropy (CWE) method given in Fig. 4.

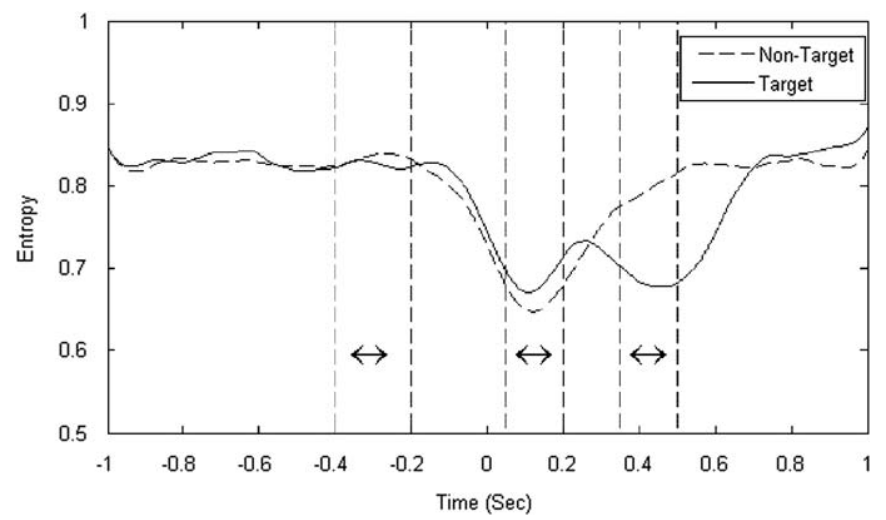

Fig. 5. The three time intervals are shown where the mean entropy values were calculated for both target (solid) and non-target (dashed) responses. The first window is in the pre-stimulus time interval $[-0.4-0.2]$ in seconds. The other time windows are chosen within the intervals [0.05 0.2] and [0.35 0.5] in seconds. One can clearly observe the difference between non-target and target patterns.

Table 1

Comparison of the continuous wavelet energy (CWE) values for the target/nontarget condition for three different time intervals.

\begin{tabular}{lll}
\hline Time $(\mathrm{s})$ & NT & $T$ \\
\hline Prestimulus $(-0.4-0.2)$ & $0.83 \pm 0.08$ & $0.83 \pm 0.08$ \\
First window $(0.050 .2)$ & $0.65 \pm 0.09$ & $0.69 \pm 0.11$ \\
Second window $(0.350 .5)^{\mathrm{a}}(0.82 \pm 0.07$ & $0.68 \pm 0.1$ \\
\hline
\end{tabular}

CWE between target and non-target data differs significantly in the time range 350-500 ms.

The mean entropy value (Shannon) provided at respected time intervals (rows). The $T$ denotes the target and NT denotes non-target cases.

${ }^{\mathrm{a}} p<0.001$.

This figure has three axes for better presentation of the evolution of single sweeps in time. The horizontal axis represents the time, whereas the vertical axis has the amplitudes. The third axis provides the formation of sweeps in time. Upon a closer look, the upper panel which demonstrates the non-target sweeps, provides a "trough" following the stimulation point (marked with "0") which is very consistent across all sweeps (towards the upper direction).

On the other hand, the target plots (lower panel) have a second "trough" around $400 \mathrm{~ms}$.

In this report, the target and non-target auditory analysis was used to perform and assess various entropy measures. The evoked potentials are responses of the brain where the brain responsiveness is time-locked to given stimuli. The auditory information processing is the key feature behind this physiological condition and responses around $50-200 \mathrm{~ms}$ are expected. However the "cognitive" component of the target responses is expected to take place at around $300 \mathrm{~ms}$. Accordingly, the brain would go from a disordered state to an ordered state for the first response. Following this, for the non-target ones the brain would shift back to disordered state. However, exceptionally for the targets, shortly after the initial ordered state formation a new ordered state is achieved, further decreasing the entropy around $300 \mathrm{~ms}$ (Fig. 5). Consequently, the results indicate this stage ordering phenomenon effectively using the proposed continuous wavelet method $(p<0.001)$. The baseline entropy was reported according to -200 to $-400 \mathrm{~ms}$ pre-stimulus interval also shown in Fig. 5.

The mean values shown in Fig. 5 related to different time intervals are illustrated in Table 1 depending on continuous time 
a

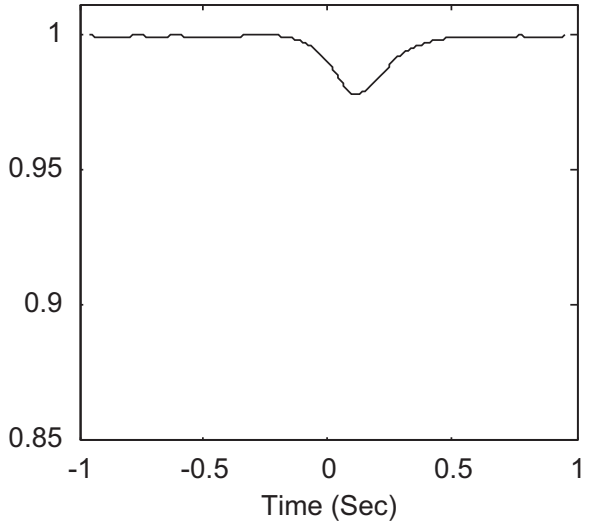

C

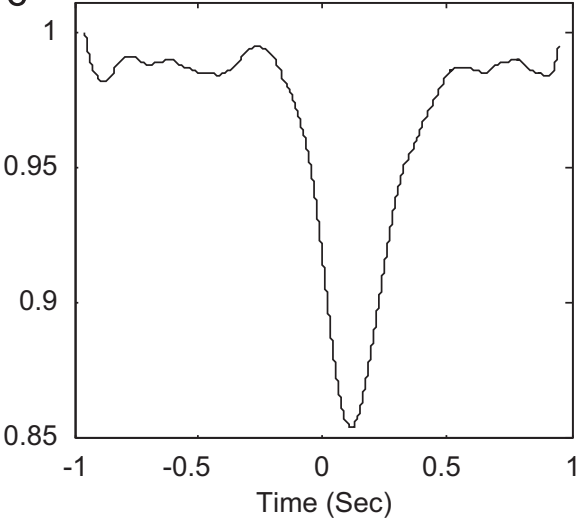

b

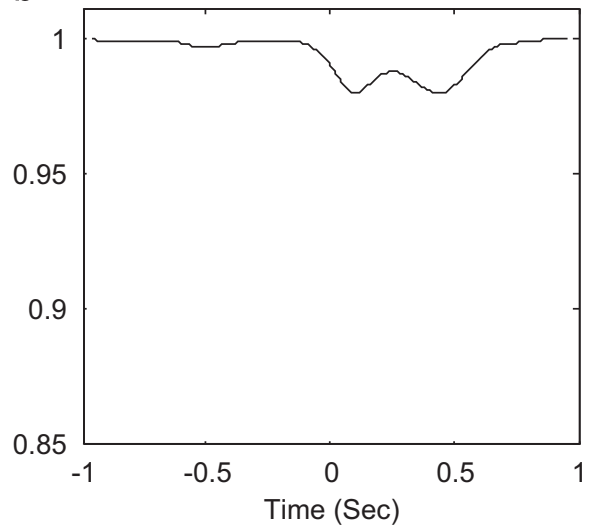

d

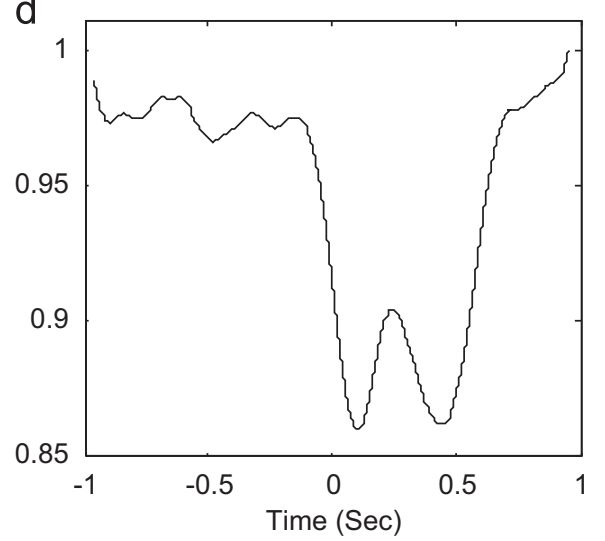

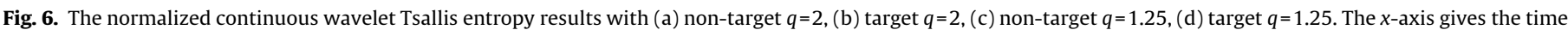
which is $1 \mathrm{~s}$ duration pre-stimulus and $1 \mathrm{~s}$ duration post-stimulus and $y$-axis gives magnitude of the normalized entropy.

wavelet entropy. It may be remarked that statistical result associated with the method applied in this work reflects the difference between non-target and targets in the second time window after stimulus. The Tsallis wavelet entropy for nontargets and targets are represented in Fig. 6 depending on different $q$ values. When the $q$ value increases, it becomes hard to observe the weak variations on wavelet entropy.

\section{Discussion}

The present paper describes the use of continuous wavelet entropy for analysis of auditory evoked potentials. Two entropy methods were applied to the same data set as Shannon and Tsallis entropy. The electrophysiological data were consisted of a prestimulus section where no brain response was expected. The stimulus was expected to force the system (brain) into an ordered state after a certain time (non-target stimulation). In the case of target stimulation a second time window following the primary response was expected to present an interesting phenomenon where a temporary disorder would be followed by an ordered state. This matches to classical P-300 response which denotes to attentional processes where the responsiveness of the brain mentally tuned to the target [11]. In this paper, we compare these two responses and the $T$-test was effectively performed for this cognitive task on the EEG data. The temporal resolution allowed reliable analysis and assessment. In comparison to "segmented" discrete analysis the temporal resolution is of a crucial issue investigating the cognitive functions with large variations and dynamic changes. Furthermore, while achieving the Shannon entropy, the wavelet coefficients were used to obtain relative wavelet energy for different frequency bands. This latter data set would be used for the oscillatory analysis of EEG activities.

The transition between ordered and disordered states characterized by the Shannon entropy was also compared by wavelet Tsallis entropy. The Tsallis entropy evaluated by wavelet coefficients was analyzed for EEG signals in several works [24-26]. Though Tsallis entropy is also a viable method when applied within the scope of continuous concept, it may suffer from the $q$ index parameter. It is observed that the increment in $q$ index causes the normalized scale to be restricted in a narrow range when Shannon and Tsallis entropies are compared in the same interval.

It may be clearly seen that the $q$ parameter of Tsallis entropy affects the range of entropy results. The increase of the $q$ parameters causes the Tsallis entropy range to get narrow and to decreases entropy resolution. The high variations are dominated while the small local entropy changes are not properly represented. When the $q$ parameter decreases then the entropy range and resolution starts to converge to the results found by Shannon entropy.

In the paper [11], it was stated that single sweep analysis would reveal additional information more than the averaged waveforms. The current report, utilized a method that is capable of analyzing single sweeps as clearly shown in Fig. 4. Here, it may be observed that even in single sweeps the event related entropy changes are achievable indicating a strong support for ordered/ disordered microstates.

According to the illustration of the Shannon wavelet entropy with respect to time, it can be easily observed that the total 
entropy decreases in post-stimulus region. Wavelet energy associated with theta and delta bands has a meaningful increase, as expected.

Thus, the normalized Shannon wavelet entropy by the means of continuous wavelet transform coefficients were applied and used in this paper. From a broader aspect, the overall entropy analysis is similar to the work in [15]. However, this current report focuses on obtaining wavelet entropy by continuous wavelet transform coefficients instead discrete wavelet based decomposition. In the literature, although the continuous wavelet transform was also used for determining wavelet entropy in $[18,19]$, these studies are related to cardiac data which are very different to the complex EEG data analysis. Additionally in papers $[15,17]$ the presented data also show decreased entropy in the post-stimulus section, confirming our findings of entropy change indicating an ordered state.

The study has some possible limitations due subject number and sweep samples. The current analysis would benefit from further applications to various biological signals and other evoked or event-related potentials. This would provide a further support for the method or it could present the areas where the method and the applications could be improved.

\section{Conclusion}

The main objective of using continuous wavelet transform is to detect the transition from the disordered to ordered state with a minimum loss of information. While analyzing the wavelet entropies of the given auditory evoked potentials, it was observed that continuous wavelet entropy could also reveal the transition to the ordered state from disordered state between 350 and $500 \mathrm{~ms}$ after the stimulus for the target EPs, whereas the discretetime non-overlapping wavelet entropy method was missing abrupt changes unless the window size is sufficiently small. Accordingly, non-continuous methods may fail to show high temporal variations. Furthermore, the discrete time entropy methods rely largely on the optimal value for the window size, which depends on the application type. However, the continuous time wavelet entropy gives detailed information about the underlying dynamical structure of the signal without similar dependence on such parameters, marking to its plasticity as a stand-alone method.

\section{Conflict of interest statement}

None declared.

\section{Acknowledgment}

We would like to thank to Onur Bayazit for his support of data preparation.

\section{References}

[1] S. Mallat, A Wavelet Tour of Signal Processing, Academic Press, New York, 1998.

[2] C.K. Chui, Wavelet Analysis and its Applications, Academic Press, New York, 1992

[3] W. Klimesch, Memory processes, brain oscillations and EEG synchronization, Int. J. Psychophysiol. 24 (1996) 61-100.

[4] C.E. Powell, I.C. Perceival, A spectral entropy method for distinguishing regular and irregular motion of Hamiltonian systems, J. Phys. A 12 (1979) 2053-2071.

[5] T. Inouye, K. Shinosaki, H. Sakamoto, S. Toi, S. Ukai, A. Iyama, Y. Katzuda, M. Hirano, Quantification of EEG irregularity by use of the entropy of powe spectrum, Clin. Neurophysiol. 79 (1991) 204-210.
[6] T. Inouye, K. Shinosaki, A. Iyama, Y. Matsumoto, Localization of activated areas and directional EEG patterns during mental arithmetic, Clin. Neurophysiol. 86 (1993) 224-230.

[7] O.A. Rosso, M.T. Martin, A. Plastino, Brain electrical activity analysis using wavelet based information tools, Physica A 313 (2002) 587-608.

[8] O.A. Rosso, S. Blanco, A. Rabinowicz, Wavelet analysis of generalized tonicclonic epileptic seizures, Signal Process. 83 (2003) 1275-1289.

[9] O.A. Rosso, M.T. Martin, A. Plastino, Brain electrical activity analysis using wavelet based information tools II, Tsallis non-extensivity and complexity measurements, Physica A 320 (2003) 497-511.

[10] J. Yordanova, V. Kolev, O.A. Rosso, M. Schürmann, O.W. Sakowitz, M. Özgören, E. Bașar, Wavelet entropy analysis of event related potentials indicates modality-independent theta dominance, J. Neurosci. Methods 117 (2002) 99-109.

[11] J. Yordanova, O.A. Rosso, V. Kolev, A transient dominance of theta event related brain potential component characterizes stimulus processing in auditory odball task, Clin. Neurophysiol. 114 (2003) 529-540.

[12] O.A. Rosso, M.T. Martin, A. Figliola, K. Keller, A. Plastino, EEG analysis using wavelet-information tools, J. Neurosci. Methods 153 (2006) 163-182.

[13] I. Daubechies, Ten Lectures on Wavelets, SIAM, Philadelphia, PA, 1992

[14] P.S. Addison, The Illustrated Wavelet Transform Handbook, IOP Publishing, 2002.

[15] O.A. Rosso, S. Blanco, J. Yordanova, V. Kolev, A. Figliola, M. Schürmann, E. Basar, Wavelet entropy: a new tool for analysis of short duration brain electrical signals, J. Neurosci. Methods 105 (2001) 65-75.

[16] R.Q. Quiroga, O.A. Rosso, E. Bașar, M. Schürmann, Wavelet entropy in event related potentials: a new method shows ordering of EEG oscillations, Biol. Cybern. 84 (2001) 291-299.

[17] O.A. Rosso, Entropy changes in brain function, Int. J. Psychophysiol. 64 (2007) $75-80$.

[18] S. Bunluechokchai, M.J. English, Analysis of the high resolution ECG with the continuous wavelet transform, IEEE Comput. Cardiol. 30 (2003) 553-557.

[19] B. Natwong, P. Sooraksa, C. Pintavirooj, S. Bunluechokchai, Wavelet entropy analysis of the high resolution ECG, in: IEEE Conference on Industrial Electronic and Applications, 2006, pp. 1-4

[20] C.E. Shannon, A mathematical theory of communication, J. Bell Syst. Tech. 27 (1948) 379623.

[21] M. Unser, Spline: a perfect fit for signal and image processing, IEEE Signal Process. Mag. 16 (1999) 22-38.

[22] P. Thévenaz, T. Blue, M. Unser, Interpolation revisited, IEEE Trans. Med. Imaging 19 (2000) 739-758.

[23] A.P. Bradley, W.J. Wilson, On wavelet analysis of auditory evoked potentials, Clin. Neurophysiol. 115 (2004) 1114-1128.

[24] A. Capurro, L. Diambra, D. Lorenzo, O. Macadar, M.T. Martin, C. Mostaccio, A. Plastino, E. Rofman, M.E. Torres, J. Velluti, Tsallis entropy and cortical dynamics: the analysis of EEG signals, Physica A 257 (1998) 149-155.

[25] S. Tong, A. Bezerianos, J. Paul, Y. Zhu, N. Thakor, Nonextensive entropy measure, of EEG following brain injury from cardiac arrest, Physica A 305 (2002) 619-628.

[26] O.A. Rosso, M.T. Martin, A. Plastino, Brain electrical activity analysis using wavelet based informational tools (II): tsallis non-extensivity and complexity measures, Physica A (2003) 497-511.

M. Emre Çek graduated in Electrical and Electronics Engineering at Dokuz Eylul University in 2001 and received M.S. degree from Izmir Institute of Technology in 2004.

He has been currently Ph.D. Student in Dokuz Eylul University, department of Electrical and Electronics Engineering since 2004 and also working as a research assistant at the Department of Electrical and Electronics Engineering in Izmir Institute of Technology since 2001.

His main research interest is focused on chaotic communication, nonlinear dynamical systems, time-frequency analysis, wavelets, particle filters and biomedical signal processing including EEG analysis.

Murat Özgören is a associate professor of medicine and is the chair of Department of Biophysics, Faculty of Medicine, Dokuz Eylul University. He has received his Ph.D. in biophysics with collaboration of Ege University and University of Miami, Biomedical Engineering. He is a member of the Brain Dynamics Research Center of Dokuz Eylul University.

His research interests include applied brain biophysics, signal processing, information processing in the brain, multidisciplinary and translational studies in the cognitive science incorporating memory, sleep and pathological processes.

F. Acar Savac1 received B.S. degree in Electrical Engineering from the Middel East Technical University, Ankara, in 1979, the M.S. degree in Electrical Engineering from Bogazici University, Istanbul Technical University, Istanbul, in 1989. He worked as postdoctoral fellow in Electronics Engineering Department of Katholieke Universiteit, Leuven, Belgium in 1990-1992. 
He worked at the Electrical-Electronics Engineering Faculty of Istanbul as a research assistant during 1982-1990, as Assistant Professor in 1990-1992 and Associate Professor since 1992 till 1998. He has been working at the Department of Electrical and Electronics Engineering in Izmir Institute of Technology,
Izmir, since February 1998 as a Full Professor and as the Chairman of the department.

His research interests are linear and nonlinear system theory, chaos, artificial neural networks, fault analysis in analog circuits and signal processing. 